\title{
SOLUTIONS TO THE CHARACTERISTIC EQUATION FOR INDUSTRIAL ROBOT'S ELLIPTIC TRAJECTORIES
}

\author{
Pavol Božek, Željko Ivandić, Alexander Lozhkin, Vadim Lyalin, Vladimir Tarasov
}

Original scientific paper

Linear independent non-projective transformations are used for different types of applications. Direct analytical method for solving the linear transformation of the characteristic equation for industrial robot is proposed. The theoretical basis of the method derives from the classical solutions with symmetries, but the method does not contain formulas with radicals. Automorphisms are divided into set theory and universal algebra. This feature allows us to use this method more efficiently for other engineering tasks.

Keywords: Diuedonne symmetries, kinematic scheme, linear transformations, industrial robot, set theory, universal algebra

Rješenja karakteristične jednadžbe za eliptične putanje industrijskog robota

Izvorni znanstveni članak

Linearne nezavisne neprojektivne transformacije rabe se za različite vrste aplikacija. Predlaže se direktna analitička metoda za rješenje linearne transformacije karakteristične jednadžbe za industrijskog robota. Teorijska osnova metode proizlazi iz klasičnih rješenja sa simetrijama, ali metoda ne sadržava formule s korijenima. Automorfizmi se dijele na teoriju skupova i opću algebru. To nam omogućuje učinkovitiju primjenu te metode za druge tehničke zadatke.

Ključne riječi: Diuedonne simetrije, kinematička shema, linearne transformacije, teorija skupova, opća algebra

\section{Introduction}

Models of height accuracy are used in CAD/CAM, space research, pattern recognition, robotics, etc. The transfer of models to nano-level cannot be provided by approximate methods. The focus of attention should be paid to research in the Euclidean plane $\boldsymbol{R} \times \boldsymbol{R}$ for reflections $\boldsymbol{R} \times \boldsymbol{R} \rightarrow \boldsymbol{R} \times \boldsymbol{R}$. The main question is how incremental parts of Cartesian product interacts with the conducts of continuity. Whilst formulating axioms for Euclidean plane $\boldsymbol{R} \times \boldsymbol{R}$, Gilbert has suggested that the construction of linguistic rules must be considered. We retain the Euclidean plane as text.

\section{Semiotic analysis of machine building drawing}

The authors have encountered some problems verifying the vector geometric model after the input from the digitizer in the early $80 \mathrm{~s}$. There was a suggestion to consider a drawing for the machine building as text. V. A. Zvyagintsev proposed the levels of research of natural language in linguistic semantics within classical linguistics. He singled out seven levels [1]: a sentence, a phrase (syntagma), a word, a morpheme, a syllable, a phoneme, and a distinctive feature. The text (discourse) is included as the eighth additional synthesis level. The second most important result of this research is the idea of binary structure of study levels. The basic structure of the language for the drawing of the machine building was proposed on the Zvyagintsev's basis [14]: a drawing, form, a cut, a geometric shape, a point, a hypercomplex number or scalar. The geometry and pattern recognition theory can alternate in the structure.

The method of semiotic analysis by A. P. Ershov interpretation has been chosen to solve the problems of verification. According to the authors, it is the most powerful method of artificial intelligence. The method is divided into three components [13]: syntax, semantics and pragmatics. Syntax is commonly referred to in the study of connection levels (morpheme, word, sentence, etc.). Semantics studies the relationship between the structure of different levels. The construction of language with the subject of the text is called pragmatics. All sections are combined in semiotics. An additional level is considered in natural language and theory of programming languages [2].

The rule has been created to resolve uncertainties [14] of the study structure. Each level of study with a number semantically related to the level $n-2$ and $n+2$ is a rule of relationships between levels. The law applies to artificial intelligence and could not be proved in the last century. This rule and semiotic analysis allowed both - to conduct research in semiotics of language drawings and to solve the problems of clarifying the geometric model.

The solution could be found using two theories: $\boldsymbol{K}_{1}^{\prime}$ and $\boldsymbol{K}_{2}^{\prime}$. Theory $\boldsymbol{K}_{1}^{\prime}$ resulted from study levels $\boldsymbol{L}_{i j}$, when $i$ - is the number of theory, $j$ - is the routine number of study levels. The study levels may be part of different mathematical descriptions.

If any binary normalization $f$ is applied to the rule $\boldsymbol{L}_{i j}$, then $\boldsymbol{L}_{i j}^{\prime}=f\left(\boldsymbol{L}_{i j}\right)$, when $\boldsymbol{L}_{i j}^{\prime} \in B, B$ - Boolean lattice. Structural linguistics may be used for study levels (symmetries in Euclidean plane) $\boldsymbol{L}_{i j}^{\prime}$ in Zveginzev's interpretation presently. Relationships between $\boldsymbol{L}_{1 j}^{\prime}$ and $\boldsymbol{L}_{2 j}^{\prime}$ is the main question in theory $\boldsymbol{K}$.

By the analogy of H. Weyl's transfer symmetry [3] we may suggest that the study levels in theory $\boldsymbol{K}$ will be positioned by law: $\boldsymbol{L}_{11}^{\prime}, \boldsymbol{L}_{21}^{\prime}, \ldots, \boldsymbol{L}_{1 m}^{\prime}, \boldsymbol{L}_{2 m}^{\prime}$ (knowledge symmetry). Any rule $\boldsymbol{L}_{i j}$ is unique. The past experience shows that if the cardinality of set is different, then the additional rules appear in law: $\boldsymbol{L}_{11}^{\prime}, \boldsymbol{L}_{21}^{\prime}, \ldots, \boldsymbol{L}_{1 m}, \boldsymbol{L}_{2 m}^{\prime}$. 
For simplicity, we realize that the following rule conserves the power of study levels: $m\left(\boldsymbol{K}_{1}^{\prime}\right)=m\left(\boldsymbol{K}_{2}^{\prime}\right)$.

Study level $\boldsymbol{L}_{i j}$ for knowledge area $\boldsymbol{K}_{i}$ may be defined in other descriptive language. This may be distant from mathematics. Therefore, syntactic rules must be formulated:

1. Binary rule: $\boldsymbol{L}_{i j}^{\prime} \in B$;

2. Rule of definition for punctuality. For example, rule of accessories of an element in a set by Fraenkel. ZFC-axiomatics (Zermelo-Fraenkel-Curatowski) is followed on from this.

3. Implication rule: $\boldsymbol{s}_{i j k} \rightarrow \boldsymbol{s}_{i j+2 m}=1$, where semantic rule (axiom) $\boldsymbol{s}_{i j k} \in \boldsymbol{L}_{i j}^{\prime}$.

\section{Extended table of Diuedonne's symmetries}

The chain of linear transformations in homogeneous coordinates was used for solutions for ellipses intersection points. There are two existing ellipses $E_{i}$ : $E_{i} \equiv<x_{i}, y_{i}, a_{i}, b_{i}, \varphi_{i}>$, where $i \in\{1,2\}, \quad\left(x_{i}, y_{i}\right) \quad$ coordinates of center ellipse, $a_{i}, b_{i}$ - semi axis, $\varphi_{i}$ - angle of rotation, $x_{i}, y_{i}, a_{i}, b_{i}, \varphi_{i} \in \boldsymbol{R}, \varphi_{i} \in[-\pi, \pi]$. The problem in classical research is solved by successive application of the methods of Descartes-Euler (fourth degree equation) and Cardano-Tartaglia (tree degree equation). The solutions are obtained with complex component: $x_{n}=x_{n R}+i x_{n C}$, where $n$ - the number of roots, $x_{n R}, x_{n C} \in \boldsymbol{R}$ in some cases. The values $x_{n R}$ and $x_{n C}$ are comparable. Finding solutions is often difficult. The analytical solution needs to involve the calculation of the intersection points. To solve the problem the authors had to turn to the theory of analytic geometry.

Hilbert, formulating axioms for Euclidean plane, suggested that the construction of linguistic rules must be considered. According to Leibniz's method of similarity, we obtain the Euclidean plane as a text by comparing semiotic analysis of drawings,. The research levels of the text are internal relations to the plane [4]. The basic information has been taken from unitary matrix symmetries by Dieudonne and Zolotova [5]; then the table automorphisms and transfer symmetry by H. Weyl [3]; definition of symmetry by M. Born [15] and binary automorphisms by F. Bachman [6].

Cartesian product was studied within the limits of ZFset theory (Zermelo-Fraenkel) because set $\{\langle x, y\rangle, x\}$ created asymmetry in $\boldsymbol{R}^{2}$. The relational algebra by $E$. Codd used Cartesian product [7]. This theory was used in many applications, but this hypothesis is in conflict with ZF-theory by primary property: connections by Codd and set by $A$. Fraenkel. ZF-theory was built from automorphic rule $a \in A$ [8]. This rule is careless without relation $\in$. The relational algebra is not used if $A \equiv \varnothing$. Therefore, relational algebra is a part of ZF-theory.

The main attention of research was devoted to the permutation symmetry $[9,10]$. The Euclidean plane is a relation table. The proof is obvious because the relation algebra may work with finite and indefinite tables. The conduct of symmetry was considered by relation of algebra and semiotic analysis. The application of the method to the Dieudonne automorphisms shows that binary symmetry belongs to two mathematical disciplines: set theory and universal algebra.

The extended table of Dieudonne symmetries was built on the basis of knowledge symmetry and relational algebra:

1. Existence of set ( $A \neq \varnothing$ Zermelo).

2. Existence of relation ( $a_{1} R a_{2}$ Codd).

3. Membership element of set ( $a \in A$ Fraenkel).

4. Universal relation ( $f: \Omega \rightarrow \Omega^{\prime}$ implication).

5. Saving cardinality $(\boldsymbol{m}(A)=$ const Lagrange $)$.

6. Saving power relations ( $n=$ const in $C_{1} x^{n}+C_{2} y^{n}+C_{3} x^{n-1} y^{n-1}+\ldots .+C_{k-1} x+C_{k} y+C_{0}$ Klein).

7. Linguistic order $\left(a_{i} \neq a_{i+1}\right.$ Descartes).

8. Mathematical order $\left(a_{i} \prec a_{i+1}\right.$, where $a_{i}, a_{i+1} \in \boldsymbol{R}$ Kantor).

9. Permutation $\left(a_{i} \leftrightarrow a_{j}\right)$.

10. Mirror $\left(a_{i} \bullet-1=-a_{i}\right)$.

Since the connection between automorphisms 8 and 10 first was suggested by Gilbert, the symmetry of knowledge might be named after this author. Dedekind's axiom called Klein automorphism, as the inverse element axiom was determined by mirror symmetry. Symmetry with numbers 1 and 2 is combined $\left(\begin{array}{ll}1 & 0 \\ 0 & 1\end{array}\right)$.

The rule of interactions of automorphisms is any relationship mapping the function, operation, operator and transformation performed in the Euclidean plane so that the performed symmetry with minor number of possible preservation of symmetries follows it. Inductive method can easily prove the sufficiency of the hierarchy in the table. The interpretation of geometry was called informatics-linguistic because it is founded on linguistic and relational algebra.

If Klein's quadratic form is [11], then $A x^{2}+2 B x y+C y^{2}+D x t+E y t+F t^{2}=0$. The parameter $t$ assumes a geometrical significance if it has been interpreted as a trace of Linguistic order symmetry. The matrix of transformation in heterogeneous coordinates is the object of set theory, therefore, the algebraic operations are not possible.

\section{The non projective methods of linear transformations of central-symmetrical conic sections}

We suggest that angle $\alpha$ exists for the quadratic form. This angle forms angle $\beta$ by the permutation symmetry. The angles are the definite non-orthogonal basis $[13,14]$. The centrally symmetric conic section with a parametric system of equations is $\left\{\begin{array}{l}x=k_{x} f_{x}(t) \\ y=k_{y} f_{y}(t)\end{array}\right.$, where $k_{x}, k_{y}, x, y, t \in \boldsymbol{R}$. The conic section is changed by linear transformation $\left(\begin{array}{ll}a & h \\ g & b\end{array}\right)$, where $a, b, g, h \in \boldsymbol{R}$. We must solve the characteristic equation $T z=\lambda z \quad[11,12]$, 
where $z=\left(\begin{array}{l}x \\ y\end{array}\right)$. The resulting curves are obtained by direct analytical method of linear transformations (DAM) of central-symmetrical conic sections (CSCS) with the following parameters:

$\tan 2 \alpha=\frac{2(b h+a g)}{\left(a^{2}+h^{2}\right)-\left(b^{2}+g^{2}\right)}$,

from classical method [12],

$\tan 2 \beta=\frac{2(a h+b g)}{\left(\left(a^{2}-h^{2}\right)-\left(b^{2}-g^{2}\right)\right)}$,

$\tan \alpha_{1}=\frac{a \sin \beta-h \cos \beta}{b \cos \beta-g \sin \beta}$

and

$\tan \alpha_{2}=\frac{b \sin \beta+g \cos \beta}{a \cos \beta+h \sin \beta}$

$\tilde{k}_{x}=\frac{k_{x}(a \cos \beta+h \sin \beta)}{\cos \alpha}$

and

$\widetilde{k}_{x}=\frac{k_{x}(b \sin \beta+g \cos \beta)}{\sin \alpha}$,

$\tilde{k}_{y}=\frac{k_{y}(a \sin \beta-h \cos \beta)}{\sin \alpha}$

and

$\tilde{k}_{y}=\frac{k_{y}(b \cos \beta-g \sin \beta)}{\cos \alpha}$.

For example, the motion of a point is located outside the axis of the flat rod. The system of equations describing the motion of the third point is $\left\{\begin{array}{l}x=e \cos t+c \sin t \\ y=c \cos t+(d-e) \sin t\end{array}\right.$, where $d$ - crank length, $e, c-$ coordinates of the point $(x, y)$ in the coordinate system of the flat rod. The proposed solution method is $\left\{\begin{array}{l}x=(d-e+c \cot \alpha) \cos (t+\alpha) \\ y=(e-c \cot \alpha) \sin (t+\alpha)\end{array}\right.$

and $\left\{\begin{array}{l}x=(e+c \tan \alpha) \cos (t+\alpha) \\ y=(d-e-c \tan \alpha) \sin (t+\alpha)\end{array}\right.$

where $\tan 2 \alpha=\frac{2 c}{(2 e-d)}$.

Solution classical method for the transformation matrix $\boldsymbol{T}=\left(\begin{array}{cc}e & c \\ c & d-e\end{array}\right)$ by the inverse matrix

$\boldsymbol{T}^{-1}=\frac{1}{\operatorname{det} \boldsymbol{T}}\left(\begin{array}{cc}d-e & -c \\ -c & e\end{array}\right)$ is $\left\{\begin{array}{l}x=\lambda_{1} \cos (t+\alpha) \\ y=\lambda_{2} \sin (t+\alpha)\end{array}\right.$ or

$\left\{\begin{array}{c}x=\lambda_{2} \cos (t+\alpha) \\ y=\lambda_{1} \sin (t+\alpha)\end{array}\right.$, where

$\lambda_{1,2}=\frac{d \pm \sqrt{d^{2}-4\left((d-e) e-c^{2}\right)}}{2\left((d-e) e-c^{2}\right)}$. Angle $\alpha$ is not

changed.

As can be seen from the results, the proposed method can be used in subsequent calculations easily, for example, to solve differential equations.

\section{Theoretical tests}

We consider the adequacy of all the theorems only.

Theorem 1: (Angles $\alpha_{1,2}$ ) Angles $\alpha_{1}$ (3) and $\alpha_{2}$ (4) is equivalence.

Proof: We compare the formula (3) and (4) in value $\tan \alpha$ :

$$
\frac{b \tan \beta+g}{a+h \tan \beta}=\frac{a \tan \beta-h}{b-g \tan \beta},
$$

$(b \tan \beta+g)(b-g \tan \beta)=(a \tan \beta-h)(a+h \tan \beta) \quad$ or $v_{1}=v_{2}$. Let's show $v_{1}=(b \tan \beta+g)(b-g \tan \beta)$ : $v_{1}=b^{2} \tan \beta+g b-g b \tan ^{2} \beta-g^{2} \tan \beta$,

$v_{1}=\left(b^{2}-g^{2}\right) \tan \beta+g b\left(1-\tan ^{2} \beta\right)$. The value $v_{2}=(a \tan \beta-h)(a+h \tan \beta) \quad$ is determinated: $v_{2}=a^{2} \tan \beta-h a+a h \tan ^{2} \beta-h^{2} \tan \beta$, $v_{2}=\left(a^{2}-h^{2}\right) \tan \beta-h a\left(1-\tan ^{2} \beta\right)$. Results equal to $\left(a^{2}-h^{2}\right) \tan \beta-h a\left(1-\tan ^{2} \beta\right)=$ $=\left(b^{2}-g^{2}\right) \tan \beta+g b\left(1-\tan ^{2} \beta\right){ }^{\prime}$ $\left(a^{2}-h^{2}\right) \tan \beta-\left(b^{2}-g^{2}\right) \tan \beta=$ $=g b\left(1-\tan ^{2} \beta\right)+h a\left(1-\tan ^{2} \beta\right)$ $\left(a^{2}-h^{2}-b^{2}+g^{2}\right) \tan \beta=(g b+h a)\left(1-\tan ^{2} \beta\right)$, $\frac{\tan \beta}{1-\tan ^{2} \beta}=\frac{g b+h a}{a^{2}-h^{2}-b^{2}+g^{2}}$, $\frac{2 \tan \beta}{1-\tan ^{2} \beta}=\frac{2(g b+h a)}{a^{2}-h^{2}-b^{2}+g^{2}}$, $\tan 2 \beta=\frac{2(g b+h a)}{a^{2}-h^{2}-b^{2}+g^{2}}$. The resulting formula coincides with the formula (2) and hence the angle $\alpha$ is the same in both formulas.

Remark: Theorem is intended to the following linear independent transformations only: $c_{1,2}=\widetilde{k}_{x 1,2}$ and $d_{1,2}=\widetilde{k}_{y 1,2}$.

Theorem 2 (Parameters of transformation): Parameters $c_{1,2}$ when $b \sin \beta+g \cos \beta \neq 0$ and $d_{1,2}$ when $b \cos \beta-g \sin \beta \neq 0$ are equivalent.

Proof: 1. For coefficient $c$ we use an opposite value.This means that $c_{1} \neq c_{2}$ and $c_{1}, c_{2} \neq 0$, then $c_{1}-c_{2}=\Delta$ from (5) and (6):

$\frac{b \sin \beta+g \cos \beta}{\sin \alpha}=\frac{a \cos \beta+h \sin \beta}{\cos \alpha}+\Delta$,

$\frac{1}{\sin \alpha}=\frac{a \cos \beta+h \sin \beta}{\cos \alpha(b \sin \beta+g \cos \beta)}+\frac{\Delta}{b \sin \beta+g \cos \beta}$,

$\frac{\cos \alpha}{\sin \alpha}=\frac{a \cos \beta+h \sin \beta}{b \sin \beta+g \cos \beta}+\frac{\Delta \cos \alpha}{b \sin \beta+g \cos \beta}$. We have from (4) and theorem $1 \tan \alpha=\tan \alpha+\frac{\Delta \cos \alpha}{b \sin \beta+g \cos \beta}$.

Equality holds when $\cos \alpha=0$ or $\Delta=0$. Since $\cos \alpha$ in general does not equal 0 , then $\Delta=0$ and parameters are 
equivalent.

2. For coefficient $d$ we use an opposite value. If $d_{1} \neq d_{2}$ and $d_{1}, d_{2} \neq 0$, then $d_{1}-d_{2}=\Delta$ from (7) and

$$
\frac{b \cos \beta-g \sin \beta}{\cos \alpha}=\frac{a \sin \beta-h \cos \beta}{\sin \alpha}+\Delta,
$$

$\frac{1}{\cos \alpha}=\frac{a \sin \beta-h \cos \beta}{\sin \alpha(b \cos \beta-g \sin \beta)}+\frac{\Delta}{b \cos \beta-g \sin \beta}$,

$\frac{\sin \alpha}{\cos \alpha}=\frac{a \sin \beta-h \cos \beta}{b \cos \beta-g \sin \beta}+\frac{\Delta \sin \alpha}{b \cos \beta-g \sin \beta}$. We have

from (3) and theorem $1 \tan \alpha=\tan \alpha+\frac{\Delta \sin \alpha}{b \cos \beta-g \sin \beta}$.

Equality holds when $\sin \alpha=0$ or $\Delta=0$. Since $\sin \alpha$ in general does not equal 0 , then $\Delta=0$ and parameters are equivalent.

Theorem 3 (Own angle): The own angle in a classical method and direct analytical method is equivalent.

Proof: We compare the formula (3) and (4) in value $\tan \beta$. We deduce the angle $\tan \beta$ of the formula (7):

$\tan \alpha_{2}(a+h \tan \beta)=b \tan \beta+g$,

$\tan \alpha_{2} a+h \tan \alpha_{2} \tan \beta=b \tan \beta+g$,

$\tan \alpha_{2} a-g=b \tan \beta-h \tan \alpha_{2} \tan \beta$,

$\frac{\tan \alpha_{2} a-g}{b-h \tan \alpha_{2}}=\tan \beta$.

We deduce the angle $\tan \beta$ of the formula (6): $\tan \alpha_{1}=\frac{a \tan \beta-h}{b-g \tan \beta}, \quad \tan \alpha_{1}(b-g \tan \beta)=a \tan \beta-h$, $\tan \alpha_{1} b-g \tan \alpha_{1} \tan \beta=a \tan \beta-h$, $\tan \alpha_{1} b+h=a \tan \beta+g \tan \alpha_{1} \tan \beta$, $\frac{\tan \alpha_{1} b+h}{a+g \tan \alpha_{1}}=\tan \beta$.

Results equal to $\frac{\tan \alpha_{1} b+h}{a+g \tan \alpha_{1}}=\frac{\tan \alpha_{2} a-g}{b-h \tan \alpha_{2}}$, $\left(\tan \alpha_{1} b+h\right)\left(b-h \tan \alpha_{2}\right)=\left(\tan \alpha_{2} a-g\right)\left(a+g \tan \alpha_{1}\right)$. We have from theorem $1 \alpha_{1}=\alpha_{2}$, then $(b \tan \alpha+h)(b-h \tan \alpha)=(a \tan \alpha-g)(a+g \tan \alpha) \quad$ or $v_{1}=v_{2}$. Let us consider $v_{1}=(b \tan \alpha+h)(b-h \tan \alpha)$ : $v_{1}=b^{2} \tan \alpha+b h-b \tan ^{2} \alpha-h^{2} \tan \alpha$, $v_{1}=\left(b^{2}-h^{2}\right) \tan \alpha+b h\left(1-\tan ^{2} \alpha\right)$. The value $v_{2}=(a \tan \alpha-g)(a+g \tan \alpha)$

$v_{2}=a^{2} \tan \alpha-g a+a g \tan ^{2} \alpha-g^{2} \tan \alpha$,

$v_{2}=\left(a^{2}-g^{2}\right) \tan \alpha-a g\left(1-\tan ^{2} \alpha\right)$. Results equal to $\left(a^{2}-g^{2}\right) \tan \alpha-\operatorname{ag}\left(1-\tan ^{2} \alpha\right)=$

$=\left(b^{2}-h^{2}\right) \tan \alpha+b h\left(1-\tan ^{2} \alpha\right)$,

$\left(a^{2}-g^{2}\right) \tan \alpha-\left(b^{2}-h^{2}\right) \tan \alpha=$

$=a g\left(1-\tan ^{2} \alpha\right)+b h\left(1-\tan ^{2} \alpha\right)$

$\left(a^{2}-g^{2}-b^{2}+h^{2}\right) \tan \alpha=(a g+b h)\left(1-\tan ^{2} \alpha\right)$,

$\frac{\tan \alpha}{1-\tan ^{2} \alpha}=\frac{a g+b h}{a^{2}+h^{2}-g^{2}-b^{2}}$, $\tan 2 \alpha=\frac{2(a g+b h)}{a^{2}+h^{2}-g^{2}-b^{2}}$. We find (1).

Coefficients of matrix are $a=b=k, g=h=0$. Angle $\beta$ is equivalent of $\tan 2 \beta=0 / 0$. An angle is not defined. If coefficients $h$ and $g$ are of equal value $\varepsilon$, where $\varepsilon \rightarrow 0$, then $\lim _{\varepsilon \rightarrow 0} \frac{4 \times \varepsilon \times k}{0}=\tan 2 \beta, \infty=\tan 2 \beta$ and $\beta=\frac{\pi}{4}$. Angle $\alpha$ is $\tan \alpha_{1}=\frac{k \tan (\pi / 4)-0}{k-0 \tan (\pi / 4)}=1$, $\alpha_{1}=\frac{\pi}{4}$ or $\tan \alpha_{2}=\frac{k \tan (\pi / 4)+0}{k+0 \tan (\pi / 4)}=1, \quad \alpha_{2}=\frac{\pi}{4}$. Angle $\alpha$ is oriented in the same way in both formulas. Coefficients $\tilde{k}_{x 1,2}$ and $\tilde{k}_{y 1,2}$ are $\tilde{k}_{x 1}=k, \tilde{k}_{x 2}=k$, $\tilde{k}_{y 1}=k, \tilde{k}_{y 2}=k$. Thus, the formulas for converting performed in the vicinity of the transformation $\left(\begin{array}{l}x^{\prime} \\ y^{\prime}\end{array}\right)=\left(\begin{array}{ll}k & 0 \\ 0 & k\end{array}\right)\left(\begin{array}{l}x \\ y\end{array}\right)$, but do not exist for accurate conversion.

The coefficients of matrix are $a=b=\cos \varphi$, $h=-\sin \varphi$ and $g=\sin \varphi$. Angle $\beta$ is an equivalent of $\tan 2 \beta=0 / 0$. An angle is also not defined. If the coefficients of matrix are $a=b=\cos \varphi+\varepsilon$, $h=-\sin \varphi-\varepsilon$ and $g=\sin \varphi+\varepsilon$, where $\varepsilon \rightarrow 0$, then angle $\beta$ will seek, as $b^{2}=a^{2}: \lim _{\varepsilon \rightarrow 0} \frac{0}{4 \varepsilon \sin \varphi}=\tan 2 \beta$, $0=\beta$. Angle $\alpha$ is $\tan \alpha_{1}=\frac{\cos \varphi \tan 0+\sin \varphi}{\cos \varphi-\sin \varphi \tan 0}=\tan \varphi$ or $\tan \alpha_{2}=\frac{\cos \varphi \tan 0+\sin \varphi}{\cos \varphi+\tan 0(-\sin \varphi)}=\tan \varphi$. Angle $\alpha$ is oriented in the same way in both formulas. Coefficients $\tilde{k}_{x 1,2}$ and $\tilde{k}_{y 1,2}$ are $\tilde{k}_{x 1}=1, \tilde{k}_{x 2}=1, \widetilde{k}_{y 1}=1, \widetilde{k}_{y 2}=1$. Findings are consistent with the conclusion of the previous conversion.

Method cannot be used for rotation $\left(\begin{array}{cc}\cos \varphi & -\sin \varphi \\ \sin \varphi & \cos \varphi\end{array}\right)$ and scaling $\left(\begin{array}{ll}k & 0 \\ 0 & k\end{array}\right)$ and some other transformations (see Theorem 2) [10].

\section{Discontinuous behavior and conduct of continuity}

Hilbert's symmetry does not define a non-orthogonal basic changes of deformed shapes for different linear transformations. The symmetry determines only the order of automorphisms in the table if we consider the components of the symmetry as a set. The authors considered the universal relation of Euler symmetry (formula $\mathrm{e}^{i \pi}=-1$ ) in earlier publications [10, 16, 17]. The careful examination of DAM suggests that it works precisely when based on this formula, because it is founded on the use of trigonometric functions in the order of a Cartesian product. However, the inverse matrix method is based on the change of the coefficients of 
discrete transform matrix, which suggests that classical method belongs to the set theory. Thus, two main symmetries are affected by the symmetry table: Hilbert's symmetry from set theory and Euler's one from universal algebra. Symmetries correspond mainly in two methods to obtain the parameters of linear transformations [18]. The concept is not in conflict with Erlangen program.

Table 1 Distinctions between classical method and DAM

\begin{tabular}{|c|c|c|}
\hline Alternative & Classical method & DAM \\
\hline Base of method & Set theory & Universal algebra \\
\hline Part of geometry & Algebraically & Differential \\
\hline $\begin{array}{c}\text { Common } \\
\text { presentation of } \\
\text { curve for solving }\end{array}$ & $\begin{array}{l}\text { Quadratic or } \\
\text { complex form }\end{array}$ & $\begin{array}{c}\text { System of } \\
\text { parametrical } \\
\text { equations }\end{array}$ \\
\hline $\begin{array}{l}\text { Contingencies in } \\
\text { finding of CSCS } \\
\text { for linearly } \\
\text { independent } \\
\text { transformations } \\
\text { (LIT) }\end{array}$ & $\begin{array}{l}\text { Shear (in own } \\
\text { basis) }\end{array}$ & $\begin{array}{l}\text { Rotation, } \\
\text { homothety }\end{array}$ \\
\hline $\begin{array}{l}\text { Symmetries using } \\
\text { for result of CSCS }\end{array}$ & 2 & 2 \\
\hline $\begin{array}{c}\text { Computation of } \\
\text { parabola's } \\
\text { parameters }\end{array}$ & Possible & Not finding \\
\hline $\begin{array}{l}\text { Symmetries for } \\
\text { parabola's finding }\end{array}$ & 4 (in Klein's form) & 0 \\
\hline $\begin{array}{l}\text { Acceptable of LIT } \\
\text { for Jordan curves }\end{array}$ & $\begin{array}{l}\text { Rotation, } \\
\text { homothety }\end{array}$ & Three groups \\
\hline $\begin{array}{c}\text { Principles of } \\
\text { processing singular } \\
\text { transformations } \\
\text { (ST) and LIT }\end{array}$ & Various & Equal \\
\hline ST groups & 0 & 6 \\
\hline $\begin{array}{l}\text { Formula of curve } \\
\text { after equal ST for } \\
\text { other curves }\end{array}$ & Individual & Common \\
\hline
\end{tabular}

The authors studied the expression of Euler's symmetry not only for conic sections but also for more complex forms. Four groups of linear $\boldsymbol{H}_{1}=\left(\begin{array}{cc}m & -n \\ m & n\end{array}\right)$, $\boldsymbol{H}_{2}=\left(\begin{array}{cc}-m & n \\ m & n\end{array}\right), \quad \boldsymbol{H}_{3}=\left(\begin{array}{cc}k m & n \\ -k n & m\end{array}\right), \quad \boldsymbol{H}_{4}=\left(\begin{array}{cc}m & k n \\ -n & k m\end{array}\right)$, where $k, m, n \in \boldsymbol{R}$, DAM can be used for any Jordan curve, however, the method is not applicable for rotation and dilation for any curve, because they do not change the discrete structure of Euclidean plane. The main distinctions between the methods are presented in Table 1. Principal differences are shown in singularities but they are not important for technical science.

During further studies, Gerono applies a parametric system of equations $\left\{\begin{array}{l}x=\cos t \\ y=\sin 2 t\end{array}\right.$. Application of Kantor's symmetry for finding the parameters of a linear transformation does not produce results for the lemniscates [16]. Diligence of Klein's symmetry allows us to find the parameters, which do not correspond to the correct result.

The proposed theory allows the calculation of complex mechatronic systems. There is a simple kinematical mechanism built on the basis of three gears: central, moving inside the central wheel and moving outside of the central wheel (Fig. 1). While a point on the first wheel moves according to the law $\left\{\begin{array}{l}x=r \cos t \\ y=r \sin t\end{array}\right.$, the motion of the second and third wheels are defined by the systems of parametric equations $\left\{\begin{array}{l}x=r \cos ^{3} \frac{t}{4} \\ y=r \sin ^{3} \frac{t}{4}\end{array}\right.$ (asteroid), $\left\{\begin{array}{l}x=3 r \cos t-r \cos 3 t \\ y=3 r \sin t-r \sin 3 t\end{array}\right.$ (epicycloids).

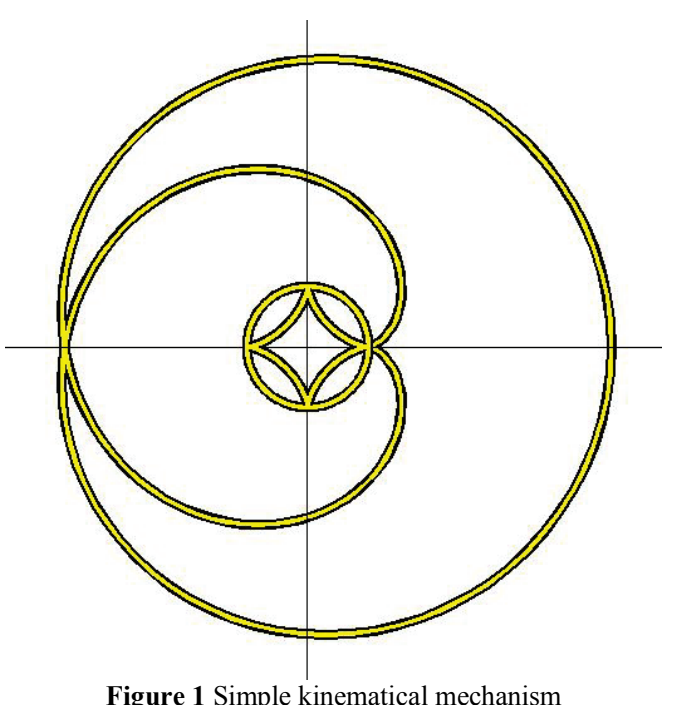

It is necessary to design an elliptical gear mechanism to the trajectory of the first wheel to a system of parametric equations $\left\{\begin{array}{l}x=-1,2 r \cos t+r \sin t \\ y=1,2 r \cos t+r \sin t\end{array}\right.$ from second group, where $m=1,2$ and $n=1$. The method of calculation can be applied to all wheels. Geometric modelling results are shown in Fig. 2.

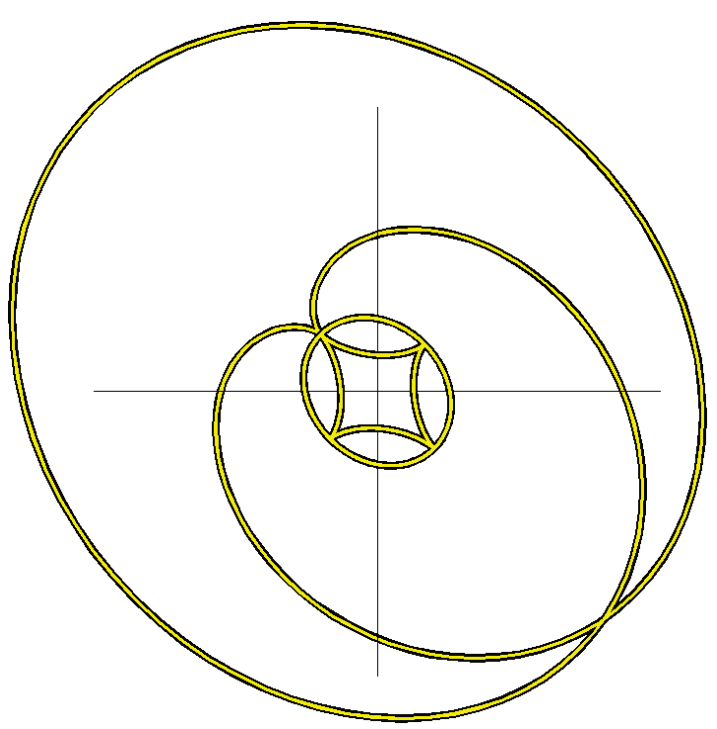

Figure 2 Complex kinematical mechanism

The program algorithm is quite simple.

An example of a geometric modelling of the method is presented to calculate the trajectories in AutoLisp AutoCAD 2009 language. 
The solution of the characteristic equation $\boldsymbol{T} \vec{v}=\lambda \vec{v}^{\prime}$ can be different. Vector $\vec{v}$ does not change after the transformation of the ellipse. Vector belongs to the set $\vec{v}^{\prime} \in\left\{\left(\begin{array}{l}x \\ y\end{array}\right),\left(\begin{array}{l}y \\ x\end{array}\right),\left(\begin{array}{l}-y \\ -x\end{array}\right)\right\} \quad$ after the transformation of the planar differentiable curve. Parameters symmetric transformations can be used to simplify the system of parametric equations for the complex movements as a result. Real coefficients can be used in systems of parametric equations. The analytical solutions of calculations are open for the complex trajectories of the mechatronic system. The precision of designing is defined within computer data storage and precise equipment. Therefore, most of the mechatronic systems can be calculated using the method proposed in this paper.

\section{Application for the design of kinematic mechanisms}

The circle and the ellipse are the basis for many of the kinematic mechanisms, such as a flat crank, crank, etc. The result of the mechanism determines the point on the trajectory $[19,20]$. The solution to differential equations can be obtained by an analytical form, if the trajectory is given of the precisely defined formula.

Rational calculation of the trajectory of motion actuators of automatic machines is important for efficient production [18]. The calculations of the motion trajectory of the kinematic mechanisms are often a result of a system of parametric equations of a circle or ellipse $\left\{\begin{array}{l}x=a \cos t+h \sin t \\ y=g \cos t+b \sin t\end{array}\right.$, where $a, b, h, g, t \in \boldsymbol{R}$.

Experiments were carried out on the basis of the developed stand to check the adequacy of the developed mathematical model. The design of the stand was assembled from aluminium profiles, linear modules and fasteners RK Rose + Krieger. A simple robot to move along the trajectory of the ellipse and the circle can be executed on the basis of the proposed mathematical model $[21,22]$. Experiments have shown a difference between the theoretical and actual trajectory of less than $5 \%$. This robot can carve with milling cutter the details in the form of an ellipse and a circle into different materials such as glass, metal, ceramics and wood. In addition, the robot can burn with laser unusual shapes in different materials.

\section{Conclusions}

Some basic properties of a described system can develop complex trajectories of Cartesian coordinates system. Along with this, there should be a similar property of the universal algebra. This may be the common property of the rings or something else. This answer could not be obtained. Tab. 1 is based on the original base research. Edgar Codd proposed thirteen rules for relational databases (Cartesian product) [17]. We used only four of them. Comparisons of Codd's rules and fundamentals of universal algebra would solve many technical problems. The wording of the missing symmetry of universal algebra can finally build an expanded table of binary Dieudonne symmetries, allowing to open a versatile method for the parameters receiving the linear transformations of Jordan curves and precise calculations used in the exact sciences such as robotics, cryptography, optimal management, etc.

\section{Acknowledgements}

The contribution is sponsored by VEGA MŠ SR No 1/0367/15 prepared project "Research and development of a new autonomous system for checking a trajectory of a robot" and project KEGA MŠ SR No 006STU-4/2015 prepared project University textbook "The means of automated production" by interactive multimedia format for STU Bratislava and Kosice".

This publication is the result of implementation of the project: "UNIVERSITY SCIENTIFIC PARK: CAMPUS MTF STU - CAMBO" (ITMS: 26220220179) supported by the Research \& Development Operational Program funded by the EFRR.

This contribution is also supported by the Operational Project Research and Development of Centre of Excellence of Five-Axis Machining, ITMS 26220120013, co-financed by the European Funds for Regional Development and supported by Minobrnauki of Russian Federation, Grant GZ/TVG 14(01.10).

\section{References}

[1] Zvegintsev, V. A. Sentence and his relation to language and speech. Moscow university, Moscow, 1976.

[2] Aho, A.V.; Lam, M. S.; Seti, R.; Ulman, J. D. Compilers. Principles, Techniques and Tools. Addison Wesley, Reading, Mass., 2007.

[3] Weyl, H. Symmetry. Princeton University Press, Cambridge, 1952. DOI: $10.1515 / 9781400874347$

[4] Lozhkin, A.; Dyukina, N. Automorphisms: from mirror to symmetry of knowledge. Lozhkin A. G., Izhevsk-Glazov, 2011.

[5] Zolotová, I.; Karch, P. Contribution to Modification of Graph Cut Method and Its Implementation in the Image Segmentation. // International Journal of Circuits, Systems and Signal Processing. 6, 1(2012), pp. 49-56.

[6] Bachman, F. Aufbau der geometrie aus dem spiegelungsbagriff. Springer-Verlag, Berlin, 1959. DOI: 10.1007/978-3-662-01234-5

[7] Codd, E. F. The Relational Model for Database Management: version 2. Addison Wesley, Reading, Mass, 1990.

[8] Fraenkel, A.; Bar-Hilel, Y. Foundations of set theory North-Holland Pub. Co, Amsterdam, 1958.

[9] Lozhkin, A. Applied planemetry with singular transformations. IE UrO RAS, Ekaterinburg, 2009.

[10] Lozhkin, A.; Dyukina, N. Structurization of analytical geometry on the base of symmetries. LAP, Saarbruken, 2012.

[11] Aleksandrov, P. Lectures on analytical geometry, supplemented by the necessary information from the algebra. Science, Moscow. 1968

[12] Efimov, N. Quadratic forms and matrices. Science, Moscow, 1967.

[13] Mathematical encyclopedic dictionary. // Ershov, A. P. Semiotic analyze, Soviet encyclopedia: Moscow, (1988), p. 845.

[14] Lozhkin, A. Method formalization of semantic relations in language of machine-building drawings. // Designing and manufacturing of metal-plastical structure, Izhevsk, IMIUdSU, (1983), pp. 81-86.

[15] Born, M. My Life and My Views. // A Nobel Prize Winner in Physics Writes Provocatively on a Wide Range of 
Subjects, NY: Scribner, (1968). pp. 63-206.

[16] Lozhkin, A. About definition of singular transformation by N. V. Efimov. // Geometry, topology, algebra and number theory, applications. In: International conference of B.N. Delone/ Steklov MI RAS, Lomonocov MSU, Moscow, (2010), pp. 160-162.

[17] Lozhkin, A. Extension of Diuedonne symmetries. // Problems of modern topology and its applications. Abst.of International conference / TSPU named after Nizami, Tashkent, (2013), pp. 56-58.

[18] Božek, P.; Turygin, Y. Measurement of the operating parameters and numerical analysis of the mechanical subsystem // Measurement Science Review. 14, 4(2014), pp. 198-203. DOI: 10.2478/msr-2014-0027

[19] Kelemen, M.; Virgala, I.; Kelemenová, T.; Miková, L.; Frankovský, P.; Lipták, T.; Lörinc, M. Distance Measurement via Using of Ultrasonic Sensor. // Journal of Automation and Control. 3, (2015), pp. 71-74.

[20] Božek, P. Robot path optimization for spot welding applications in automotive industry // Tehnički Vjesnik Technical Gazette. 20, 5(2013), pp. 913-917.

[21] Gmiterko, A.; Virgala, I. Simplified Model of the Snake Rectilinear motion. $/ / 9^{\text {th }}$ IEEE International Symposium on AMIaI, (2011), pp. 307-310.

[22] Lozhkin, A.; Abramov, I.; Bozek, P.; Nikitin, Y. The issue of calculating elliptic trajectories. // Manufacturing Technology. 14, 4(2014), pp. 561-566.

\section{Authors' addresses}

Pavol Božek, PhD. Full Prof.

Slovak University of Technology,

Faculty of Materials Science and Technology,

Institute of Applied Informatics, Automation and Mathematics,

Hajdóczyho 1, 91724 Trnava, Slovakia

Tel.: +421906 068704, pavol.bozek@stuba.sk

Željko Ivandić, PhD. Full Prof.

J. J. Strossmayer University of Osijek,

Mechanical Engineering Faculty in Slavonski Brod,

Trg Ivane Brlić-Mažuranić 2, 35000 Slavonski Brod, Croatia

Tel.: +385 35493 417, zivandic@sfsb.hr

Alexander G. Lozhkin, Doctor, Prof.

M. T. Kalashnikov Izhevsk State Technical University, Department of Software,

Studencheskaya st. 7, 426069 Izhevsk, Russia

Tel.: +7431-2-591792, lag.izh@gmail.com

Vadim Ev. Lyalin, Doctor, Prof.

Dean of the Faculty "Computer Science",

M. T. Kalashnikov Izhevsk State Technical University,

Studencheskaya st. 7, 426069 Izhevsk, Russia

Tel.: +7431-2-777739, velyalin@mail.ru

Vladimir G. Tarasov, PhD, Prof.

M. T. Kalashnikov Izhevsk State Technical University,

Department of Software,

Studencheskaya st. 7, 426069 Izhevsk, Russia

Tel.: +7909-0646347, taras_vg@mail.ru 